\title{
The Distribution of Burst Energy and Shock Parameters for Gamma-ray Bursts
}

\author{
Pawan Kumar \\ IAS, Princeton, NJ 08540
}

Received — 


\begin{abstract}
We calculate the statistical distribution of observed afterglow flux, in some fixed observed frequency band, and at some fixed observer time after the explosion $\left(t_{o b s}\right)$ in two models - one where the explosion takes place in a uniform density medium and the other where the surrounding medium has a power-law stratification such as is expected for a stellar wind. For photon energies greater than about 500 electron-volt and $t_{o b s} \gtrsim 10^{3} \mathrm{sec}$ the afterglow flux distribution functions for the uniform ISM and the wind models are nearly identical. We compare the width of the theoretical distribution with the observed x-ray afterglow flux and find that the FWHM of the distribution for energy in explosion and the fractional energy in electrons $\left(\epsilon_{e}\right)$ are each less than about one order of magnitude and the FWHM for the electron energy index is 0.6 or less.
\end{abstract}

Subject headings: gamma rays: bursts - gamma-rays: theory 


\section{Introduction}

The improvement in the determination of the angular position of gamma-ray bursts (GRBs) by the Dutch-Italian satellite Beppo/SAX has led to the discovery of extended emission in lower energy photons lasting for days to months, which has revolutionized our understanding of GRBs (cf. Costa et al. 1997, van Paradijs et al. 1997, Bond 1997, Frail et al. 1997). The afterglow emission was predicted prior to their actual discovery by a number of authors (Paczyński \& Rhoads, 1993; Meszaros \& Rees, 1993; Katz, 1994; Meszaros \& Rees 1997) based on the calculation of synchrotron emission in a relativistic external shock. The afterglow observations have been found to be in good agreement with these theoretical predictions (cf. Sari, 1997; Vietri, 1997a; Waxman, 1997; Wijers et al. 1997).

The medium surrounding the exploding object offers some clue as to the nature of the explosion. Vietri (1997b), and Chevalier and Li (1999a-b) in two very nice recent papers, have offered evidence that some GRB afterglow light curves are best explained by a stratified circumstellar medium which suggests the death of a massive object as the underlying mechanism for gamma-ray burst explosions as was suggested by Paczyński (1998), and Woosley (1993). Possible further evidence in support of such a model has come from the flattening and reddening of afterglow emission, a few days after the burst, in optical wavelength bands (eg. Bloom et al. 1999, Castro-Tirado \& Gorosabel 1999, Reichart 1999, Galama et al. 1999).

The goal of this paper is to explore the afterglow flux in different models, uniform ISM as well as stratified medium, and compare it with observations in a statistical sense, as opposed to comparison with individual GRBs as carried out by Chevalier and Li (1999b). We will use this statistical comparison to constrain various physical parameters that determine the afterglow luminosity such as the energy, $E$, the fractional energy in electrons $\left(\epsilon_{e}\right)$ and magnetic field $\left(\epsilon_{B}\right)$, the electron energy index $(p)$, and the circumstellar density $n$.

In the next section we discuss the afterglow flux and its distribution and comparison with observations.

\section{Afterglow flux and its distribution}

Consider an explosion which releases an equivalent of isotropic energy $E$ in a medium where the density varies as $A r^{-s} ; r$ is the distance from the center of the explosion, and $A$ is a constant. The deacceleration radius, $r_{d}$, where the shell starts to slow down as a result of sweeping up the circumstellar material, and the deacceleration time, $T_{d a}$, in the observer frame are given by 


$$
R_{d a}=\left[\frac{(17-4 s) E}{2 \pi c^{2} A \Gamma_{0}^{2}}\right]^{1 /(3-s)}, \quad T_{d a}=\frac{R_{d a}}{4 \beta c\left(\Gamma_{0} / 2\right)^{2}},
$$

where $\Gamma_{0}$ is the initial Lorentz factor of the ejecta, and $\beta \approx 1$ is a constant.

The time dependence of the shell radius and the Lorentz factor can be obtained from the self-similar relativistic shock solution given in Blandford \& McKee (1976)

$$
\frac{R\left(t_{o b s}\right)}{R_{d a}} \equiv X=t_{1}^{1 /(4-s)}, \quad \Gamma\left(t_{o b s}\right)=\frac{\Gamma_{0}}{2} t_{1}^{-(3-s) /(8-2 s)}, \quad \text { where } \quad t_{1} \equiv \frac{t_{o b s}}{(1+z) T_{d a}}
$$

and $t_{o b s}$ is the time in observers' frame at redshift $z$.

The magnetic field and the electron thermal Lorentz factor behind the forward shock vary as,

$$
B=B_{d a} \epsilon_{B}^{1 / 2} t_{1}^{-3 /(8-2 s)}, \quad \gamma_{e}=\epsilon_{e}\left(\frac{m_{p}}{m_{e}}\right) \frac{\Gamma}{2^{1 / 2}}, \quad \text { where } \quad B_{d a}=\left[\frac{2(17-4 s) E}{\Gamma_{0}^{2} R_{d a}^{3}}\right]^{1 / 2}
$$

and $\epsilon_{B}$ and $\epsilon_{e}$ are the fractional energies in the magnetic field and the electrons, respectively.

Using these results we find that the peak of the synchrotron frequency $\left(\nu_{m}\right)$ and the cooling frequency $\left(\nu_{c}\right)$, in the observer frame, are

$$
\nu_{m}=\nu_{m, d a} \epsilon_{e}^{2} \epsilon_{B}^{1 / 2} t_{1}^{-3 / 2}, \quad \nu_{c}=\nu_{c, d a} \epsilon_{B}^{-3 / 2} t_{1}^{(3 s-4) /(8-2 s)}
$$

where

$$
\nu_{m, d a}=\frac{1}{32 \pi 2^{1 / 2}} \frac{q B_{d a} m_{p}^{2}}{c m_{e}^{3}}, \quad \nu_{c, d a}=\frac{9 \pi}{42^{1 / 2}} \frac{m_{e} q c^{3} \Gamma_{0}^{3}}{\sigma_{T}^{2} B_{d a}^{3} R_{d a}^{2}} .
$$

The synchrotron self-absorption frequency (in the observer frame) is given by

$$
\nu_{A}=\left[\frac{27^{1 / 2} m_{e} c^{2} \sigma_{T} A R_{d a}^{1-s} B_{d a} \Gamma_{0}}{64 \pi q m_{p}^{2}}\right]^{3 / 5} \epsilon_{e}^{-3 / 5} \epsilon_{B}^{3 / 10} t_{1}^{-0.3(4+s) /(4-s)}\left[\min \left(\nu_{m}, \nu_{c}\right)\right]^{-1 / 5} .
$$

The energy flux at the peak of the spectrum is given by

$$
f_{\nu_{p}}=\frac{27^{1 / 2}}{32 \pi}\left[\frac{m_{e} \sigma_{T} c^{2}}{q m_{p} d_{L}^{2}}\right] A \Gamma_{0} B_{d a} R_{d a}^{(3-s)} \epsilon_{B}^{1 / 2}(1+z) t_{1}^{-s /(8-2 s)},
$$


where $\nu_{p}=\min \left\{\nu_{m}, \nu_{c}\right\}$ i.e. for $\nu_{m}>\nu_{c}$ the peak occurs at $\nu_{c}$ instead of at $\nu_{m}$.

The equations for $s=2$ are as in Chevalier and Li (1999a-b) and are given here for easy reference. The flux at an arbitrary observed frequency $\nu$ can be calculated following Sari, Piran and Narayan (1998) in terms of $f_{\nu_{p}}, \nu_{m}, \nu_{c}$ and $\nu_{A}$. For the particularly important case of $\nu$ greater than $\nu_{m}$ and $\nu_{c}$ the observed flux is:

$f_{\nu}=f_{\nu_{p}} \nu_{c}^{1 / 2} \nu_{m}^{(p-1) / 2} \nu^{-p / 2}=\frac{3^{2.5} c^{5}}{\nu^{p / 2} d_{L}^{2}}\left(\frac{q m_{p}^{2}}{m_{e}^{3}}\right)^{(p-2) / 2} \frac{\epsilon_{e}^{p-1}}{\epsilon_{B}^{(2-p) / 4}} \frac{(1+z)^{(3 p+2) / 4}}{t_{o b s}^{(3 p-2) / 4}}\left[\frac{(17-4 s) E}{2^{10} \pi^{2} c^{5}}\right]^{(p+2) / 4}$,

Note that the flux does not depend on the circumstellar density parameters $A$ and $s$ when $\nu>\nu_{c}$, except through an unimportant multiplicative factor $(17-4 s)^{(p+2) / 4}$.

The frequencies $\nu_{m} /\left(\epsilon_{e}^{2} \epsilon_{B}^{1 / 2}\right), \nu_{c} \epsilon_{B}^{3 / 2}$ and $\nu_{A}$ are shown in figure 1. The afterglow flux for the uniform ISM and the wind models only differ when $\nu<\nu_{c}$. Since the cooling frequency decreases with time for the uniform ISM model and increases with time for the wind model, one of the best ways to distinguish between these models is by observing the behavior of the light-curve when $\nu_{c}$ crosses the observed frequency band as has been pointed out by Chevalier \& Li (1999). The predictions and comparison of individual GRB lightcurves for the two models will be discussed in some detail in a separate paper. Here we turn our attention to the statistical property of the afterglow light curve in the two models.

\subsection{Afterglow flux distribution function}

The distribution function for GRB afterglow flux, $P_{t}\left(L_{\nu}\right)$, at a frequency $\nu$, and time

$t_{o b s, g}$ is the probability that the afterglow luminosity (isotropic) is $L_{\nu}$ at time $t_{o b s, g}$ after the explosion; $\nu, t_{o b s, g}$ and $L_{\nu}$ are measured in the rest frame of the host galaxy.

The width of $P_{t}\left(L_{\nu}\right)$ is a function of the width of the distribution function for $E, \epsilon_{e}, \epsilon_{B}$, $A$ and $p$. Assuming that all these variables are independent Gaussian random variables the standard deviation (SD) for $\log \left(L_{\nu}\right), \sigma_{L_{\nu}}$, can be obtained from equation (8), when $\nu>\nu_{c}$ $\& \nu_{m}$, and is given by

$$
\sigma_{L_{\nu}}^{2}=\left(\frac{p+2}{4}\right)^{2} \sigma_{E}^{2}+(p-1)^{2} \sigma_{\epsilon_{e}}^{2}+\eta \sigma_{p}^{2}+\left(\frac{p-2}{4}\right)^{2} \sigma_{\epsilon_{B}}^{2}
$$


where

$$
\eta=\frac{1}{16}\left[2 \log \frac{q m_{p}^{2}}{m_{e}^{3}}+\log \left(\frac{17 \bar{\epsilon}_{B} \bar{\epsilon}_{e}^{4} \bar{E}}{2^{10} \pi^{2} c^{5} \nu^{2} t_{o b s, g}^{3}}\right)\right]^{2},
$$

$\bar{\epsilon}$ and $\bar{E}$ are the mean values of $\epsilon$ and $E$, and $\sigma_{E}, \sigma_{\epsilon_{e}}, \sigma_{\epsilon_{B}}$ and $\sigma_{p}$ are the standard deviation for $\log E, \log \epsilon_{e}, \log \epsilon_{B}$ and $p$ respectively; $\eta$ for x-ray (10 kev) and optical (2.5 ev) photons are shown in figure 2 .

The standard deviation of the flux in the 2-10 kev band at 5 hours after the burst $\left(\sigma_{L_{\nu}}\right)$ is approximately 0.58 (Kumar \& Piran, 1999). Fig 1 shows that this energy band is above $\nu_{c} \& \nu_{m}$ so long as $\epsilon_{B}>10^{-4}$ and the density of the surrounding medium is not too small. Moreover, $\eta=5$ for this energy band (see fig. 2), from which we obtain an upper limit on $\sigma_{p}$ of 0.26 and the full-with at half-maximum of the distribution for $p$ to be less than about 0.6. We note that the electron energy index $p$ lies between 2 and 3 for supernovae remanents (cf. Chevalier 1990, Weiler et al. 1986), and Chevalier and Li (1999b) point out that the range in $p$ for GRB afterglows is at least $\sim 2.1-2.5$.

We can use the variation of $\eta$ with time or $\nu$ (see figs. $2 \& 3$ ) to obtain $\sigma_{p}$ from the observed variation to the width of $\sigma_{L_{\nu}}$. This is of course equivalent to the determination of $p$ from the slope of the light curve or the spectral slope. The currently available data, consisting of 7 bursts with known redshifts, does not provide a good constraint on the temporal variation of $\sigma_{L_{\nu}}$, however, HETE II and Swift would increase the GRB afterglow data base by more than an order of magnitude and provide much better constraint on $\sigma_{p}$.

Unless $\epsilon_{B}$ varies by many orders of magnitude from one burst to another the last term in equation (9) is very small and can be neglected. Equating the first two terms individually to $\sigma_{L_{\nu}}=0.58$ we obtain $\sigma_{E}<0.51$, and $\sigma_{\epsilon_{e}}<0.39$ (for $p=2.5$ ). For comparison, if the first three terms in equation (9) were to contribute equally to $\sigma_{L_{\nu}}$ then we obtain $\sigma_{E}=0.29$, $\sigma_{\epsilon_{e}}=0.22$, and $\sigma_{p}=0.15$. The mean values for $E$ and $\epsilon_{e}$ are not well determined by this procedure (but see the discussion below), however $\bar{E} \bar{\epsilon}_{e}^{4(p-1) /(p+2)}$ can be accurately obtained from the observed distribution and is $\approx 10^{52} \mathrm{erg}$.

So far we have discussed a nearly model independent procedure for determining $\sigma_{p}$ and a linear combination of $\sigma_{E}^{2}$ and $\sigma_{\epsilon_{e}}^{2}$ that relies on making observations in a frequency band that lies above $\nu_{m} \& \nu_{c}$. In order to determine $\sigma_{E}$ and $\sigma_{\epsilon_{e}}$ separately we need to have some knowledge of $\nu_{c}$ and $\nu_{m}$, and therefore the result is model dependent and less certain. For instance, if the cooling and the peak synchrotron frequencies are known at some time, even if only approximately, then $\sigma_{E}$ can be determined from the distribution of the observed flux at a frequency $\nu$ such that $\nu_{c}<\nu<\nu_{m}$. The SD for $f_{1} \equiv L_{\nu} t_{o b s, g}^{1 / 4} \nu^{1 / 2}$ at such an intermediate frequency is independent of $s$ and is given by:

$$
\sigma_{f_{1}}^{2}=\frac{9}{16} \sigma_{E}^{2}+\frac{1}{16} \sigma_{\epsilon_{B}}^{2} \approx \frac{9}{16} \sigma_{E}^{2}
$$


Once $\sigma_{E}$ is known, equation (9) can be used to determine $\sigma_{\epsilon_{e}}$. Observations at low frequencies i.e. $\nu<\nu_{m}, \nu_{c}$, can be used to constrain $\sigma_{\epsilon_{B}}$ and $\sigma_{A}$ which when combined with the flux at the peak of the spectrum could be used to determine $\sigma_{\epsilon_{B}} \& \sigma_{A}$ separately with the use of the following equations,

$$
\begin{array}{ll}
\sigma_{f_{2}}^{2}=\frac{4}{(4-s)^{2}} \sigma_{A}^{2}+\frac{4}{9}\left(\sigma_{\epsilon_{e}}^{2}+\sigma_{\epsilon_{B}}^{2}\right)+\left(\frac{14-5 s}{12-3 s}\right)^{2} \sigma_{E}^{2}, & \text { for } \quad \nu<\nu_{m}<\nu_{c} \\
\sigma_{f_{3}}^{2}=\frac{4}{9(4-s)^{2}} \sigma_{A}^{2}+\sigma_{\epsilon_{B}}^{2}+\left(\frac{14-6 s}{12-3 s}\right)^{2} \sigma_{E}^{2}, & \text { for } \quad \nu<\nu_{c}<\nu_{m},
\end{array}
$$

and

$$
\sigma_{f_{4}}^{2}=\frac{1}{4} \sigma_{\epsilon_{B}}^{2}+\frac{4}{(4-s)^{2}} \sigma_{A}^{2}+\left(\frac{8-3 s}{8-2 s}\right)^{2} \sigma_{E}^{2}
$$

where $f_{1} \equiv \nu^{-1 / 3} L_{\nu} t_{o b s, g}^{(s-2) /(4-s)}, f_{2} \equiv \nu^{-1 / 3} L_{\nu} t_{o b s, g}^{(3 s-2) /(12-3 s)}, f_{3} \equiv L_{\nu_{p}} t_{o b s, g}^{s /(8-2 s)}$, and $L_{\nu_{p}}$ is the isotropic luminosity at the peak of the spectrum.

Another approach to determining the burst and shock parameters is to compare the observed flux distributions at several different frequencies and time with the theoretically calculated distributions. The latter can be easily calculated by varying $E, \epsilon_{e}, \epsilon_{B}, A$ and $p$ randomly and solving for the flux using the equations given in the last section. Fig. 3 shows a few cases of flux distribution functions for several different $\nu$ and $t_{o b s}$. The advantage of this procedure is that it does not require observational determination of various characteristic frequencies i.e. $\nu_{m}, \nu_{c}$, and $\nu_{A}$, which is difficult to do unless we have good spectral and temporal coverage over many orders of magnitude. Moreover, this procedure can be used even without the knowledge of burst redshifts; the redshift distribution of a representative sub-sample of bursts can be used to calculate the expected theoretical distribution of observed afterglow flux which can be directly compared with the observed flux distribution to yield the burst and shock parameters.

It should be noted that if $\nu_{m}, \nu_{c}, \nu_{A}$ and $L_{\nu_{p}}$ can be determined accurately then $E, \epsilon_{e}$, $\epsilon_{B}$, and $A$ can be obtained for individual bursts as described in eg. Chevalier \& Li (1999b), and Wijers \& Galama (1999), and there is no need to resort to the statistical treatment discussed above. 


\section{Conclusion}

We have described how the distribution function for GRB afterglow flux can be used to determine the width of the distribution function for the energy in the explosion and the shock parameters such as $\epsilon_{e}, \epsilon_{B}$ (the fractional energy in electrons and the magnetic field), $p$ (the power law index for electron energy), and $A$ (the interstellar density parameter).

The afterglow flux at a frequency above the cooling and the synchrotron peak frequencies is independent of interstellar density and scales as $E^{(p+2) / 4} \epsilon_{e}^{(p-1)} \epsilon_{B}^{(p-2) / 4}$ for uniform ISM as well as for energy deposited in a stellar wind with power-law density stratification. Using the flux in 2-10 kev band for 7 bursts with known redshifts, 5 hours after the burst, which meet the above criteria, we find that the full width at half maximum of the distribution for $\log E$ is less than 1.2, for $\log \epsilon_{e}$ is less than 0.9 and $p$ is less than 0.6. The width for the distribution of $p$ is consistent with the range in $p$ deduced from afterglow emissions (cf. Chevalier \& Li, 1999b). The width of the distribution for $p$ can be more accurately determined from the time variation, or frequency dependence, of the width of the afterglow flux distribution.

For a more accurate determination of the distribution of other parameters we need to determine the cooling and the synchrotron peak frequencies (at least approximately), or otherwise compare the theoretical and the observed distributions for flux at several different frequencies covering the range above and below the cooling and the synchrotron frequencies.

HETE II and Swift missions are expected to significantly increase the number of GRBs with observed afterglow emission and should therefore provide a more accurate determination of afterglow luminosity function and the distribution for burst energy and shock parameters.

Acknowledgment: I am indebted to Roger Chevalier for many useful discussions and for clarifying several points. I thank Alin Panaitescu, Tsvi Piran and Bohdan Paczyński for numerous exciting discussions about gamma-ray bursts, and Eliot Quataert for comments on the paper. I thank E. Waxman for sending me his recent preprint which bears some technical similarity with this work, although the results and conclusions are different. 


\section{REFERENCES}

Blandford, R.D., and McKee, C.F., 1976, Phys. Fluids, 19, 1130

Bloom, J.S., et al., 1999, Nature 401, 453

Bond, H.E. 1997, IAU Circ. 6654

Catro-Tirado, A., and Gorosabel, J., 1999, A\&AS 138, 449

Chevalier, R.A., 1990, in Supernovae, A.G. Petschek (ed.), Springer-Verlag

Chevalier, R.A., and Li, Z-Y, 1999a, 520, L29

Chevalier, R.A., and Li, Z-Y, 1999b, astro-ph/9908272

Costa, E., et al. 1997, IAU Circ. 6572

Frail, D.A. et al. 1997, Nature 389, 261

Galama, T.J., et al., 1999, astro-ph/9907264

Katz, J.I., 1994, ApJ 432, L107

Mészáros, P., and Rees, M.J., 1993, ApJ 405, 278

Mészáros, P., and Rees, M.J., 1997, ApJ 476, 232

Paczyński, B., 1998, ApJ 494, L45

Paczyński, B., and Rhoads, J., 1993, ApJ 418, L5

Reichart, D.E., 1999, ApJ 521, L111

Sari, R., 1997, ApJ 489, L37

Sari, R., \& Piran, T. \& Narayan, R. 1998, ApJ 497, L17

van Paradijs, J., et. al. 1997, Nature 386, 686

Vietri, M., 1997a, ApJ 488, L33

Vietri, M., 1997b, ApJ 488, L105

Waxman, E., 1997, ApJ 489, L33

Weiler, K.W., Sramek, R.A., Panagia, N. van der Hulst, J.M., and Salvati, M., 1986, ApJ 301, 790

Wijers, R.A.M.J., and Galama, T.J., 1999, ApJ 523, 177

Wijers, R.A.M.J., Rees, M.J., \& Mészáros, P., 1997, MNRAS 288, 51

Woosley, S.E., 1993, ApJ 405, 273 




Fig. 1. - The figure shows several different frequencies, in electron volts, as a function time in observer frame. The solid line is the peak synchrotron frequency for $\epsilon_{B}=1 \& \epsilon_{e}=1$ for the wind model i.e. $\nu_{m} /\left(\epsilon_{B}^{1 / 2} \epsilon_{e}^{2}\right)$ for $s=0 ; \nu_{m}$ for uniform interstellar medium is larger by a constant factor of 1.374. The dotted curve is the cooling frequency, $\nu_{c} \epsilon_{B}^{3 / 2} n_{0}$, for uniform ISM ( $n_{0}$ is particle number density in the ISM). The dashed curve is $\nu_{c} \epsilon_{B}^{3 / 2} A_{*}^{2}$ for the wind model $\left(A_{*}\right.$ is the baryon density in the wind in the units of $5 \times 10^{11} \mathrm{~g} \mathrm{~cm}^{-1}$ ). The energy in the explosion $E$ has been taken to be $10^{52}$ erg and $p=2.5$. 


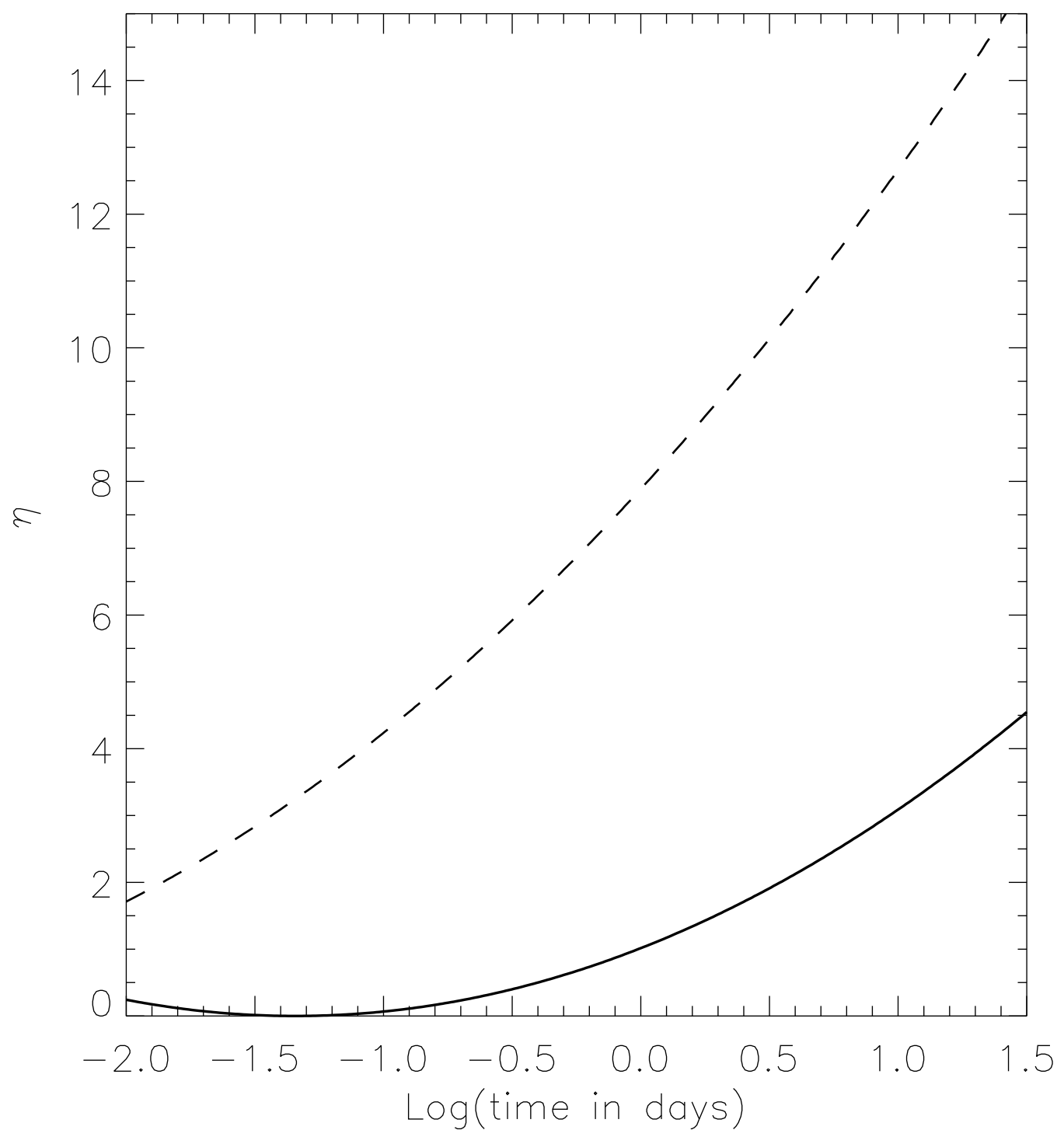

Fig. 2.- $\eta$ as defined in equation (10) is shown as a function of time for two different frequencies $-2.5 \mathrm{ev}$ (solid curve) and $10 \mathrm{kev}$ (dashed curve). We took $E=10^{52} \mathrm{erg}$, $\bar{\epsilon}_{e}=0.1$, and $\bar{\epsilon}_{B}=0.03$ for these calculations. 

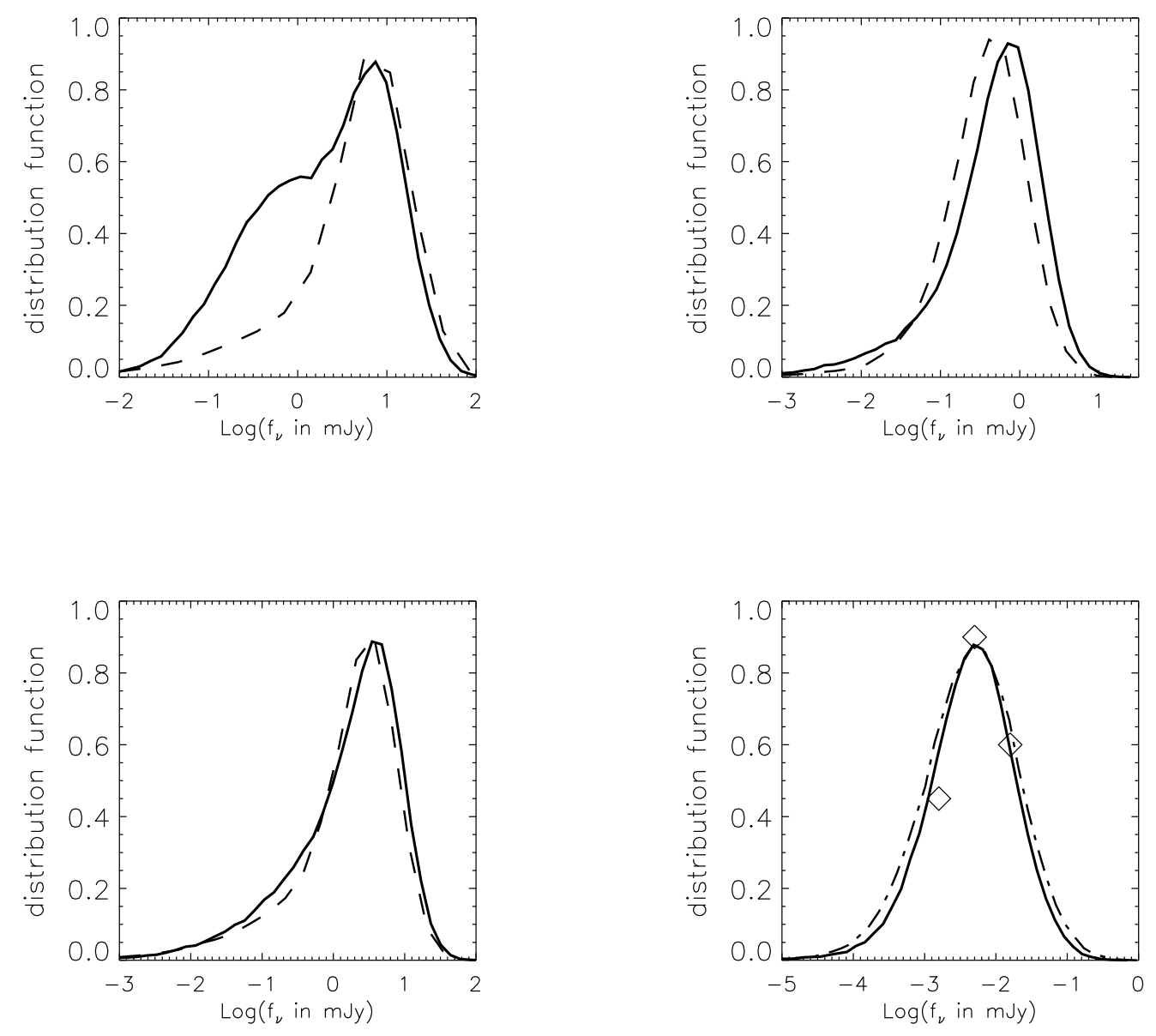

Fig. 3.- Distribution function for afterglow flux per unit frequency at photon energy of 1.0 ev, $0.1 \mathrm{hr}$ after the burst (top left panel), for uniform ISM (solid line) and stratified medium with $s=2$ (dashed line). The top right panel shows the DFs at photon energy of $100 \mathrm{ev}$ and at $0.1 \mathrm{hr}$ after the burst; solid line is for ISM and dashed line is for $s=2$. The lower left panel shows DFs for photon energy of $1 \mathrm{ev}$ at $1 \mathrm{hr}$ after the burst; solid line is for ISM and dashed line is for $s=2$. The lower right panel shows the DFs for $10 \mathrm{kev}$ energy photons at $0.1 \mathrm{hr}$ after the burst (solid line) and at $10 \mathrm{hr}$ (dash-dot curve) for the uniform ISM model; the dash-dot curve has been shifted to the right by 2.4. The DFs for the wind model $(s=2)$ for $10 \mathrm{kev}$ energy band are identical to the uniform ISM DFs at times greater than $0.5 \mathrm{hr}$ except for an overall shift of the curve to lower energy by a factor of $\sim 2$. For all of these calculations the FWHM of $\log E, \log \epsilon_{e}, \log \epsilon_{B}, A$ and $p$ was taken to be $0.8,0.7,3.0,2.4$, and 0.25 respectively, and the burst redshift was taken to be 1.5 . Moreover, $\bar{E}=10^{52} \mathrm{erg}$, $\bar{\epsilon}_{e}=0.1, \bar{\epsilon}_{B}=0.01$, and $\bar{p}=2.3$. The diamonds in the lower panel are for bursts with known redshifts; the error bar associated with each point is much larger than the size of the symbol. 